\title{
ON ORDINALLY CLOSED SETS
}

\author{
JOHN C. MORGAN II
}

\begin{abstract}
Extensions of Cantor's Intersection Theorem and Zalcwasser's theorem on transfinite sequences of ambiguous sets of the first Baire class are given for linear sets.
\end{abstract}

All sets considered in this article are assumed to be subsets of the real line $X$. The symbol $\omega$ denotes the first infinite ordinal number and $\Omega$ denotes the first uncountable ordinal number.

1. Descending sequences of sets. Let $E_{0} \supset E_{1} \supset \ldots \supset E_{n} \supset \ldots$ be a descending sequence of subsets of $X$. If each $E_{n}$ is a bounded, closed interval, then, as shown by Cantor, the intersection of the sets is nonempty. The conclusion continues to hold if the sets are only assumed to be nonempty compact sets. This latter fact, although commonly referred to as the Cantor Intersection Theorem, appears to have been first established by R. Baire (see $[1$, p. 48], and [4, p. 127]).

In [5] (see p. 14), an extension of Cantor's theorem for intervals was given where the sets are only assumed to have the same order type as a bounded, closed interval; i.e., which have the order type $1+\lambda+1$, where $\lambda$ denotes the order type of the real line $X$.

Upon modification of the proof in [5], we obtain in this section a further extension which contains all three of the preceding results.

Definition 1. A set $E$ is called ordinally closed if $E$ is order isomorphic to a closed set, and is called ordinally compact if it is order isomorphic to a compact set.

Equivalently, $E$ is ordinally closed if and only if $E$, with the relativized ordering of $X$, is a conditionally complete lattice; and $E$ is ordinally compact if and only if, in the relativized ordering, $E$ is a complete lattice.

For a discussion of ordinally closed sets, see [2, pp. 128-136], [11, pp. 132-134], and [3, pp. 17-43] (especially Théorème V, p. 42, which establishes the fact that a complete lattice $E$ in $X$ is an ordinally compact set).

THEOREM 1. The intersection of a descending sequence $\left\langle E_{n}\right\rangle_{n<\omega}$ of nonempty ordinally compact sets is nonempty.

Received by the editors October 26, 1976 and, in revised form, May 31, 1977.

AMS (MOS) subject classifications (1970). Primary 04A15, 06A05.

Key words and phrases. Cantor's Intersection Theorem, ordinally closed sets, stationary sequences of sets, monotone family of sets. 
Proof. Assuming the conclusion does not hold, we shall establish the existence of a subset $\left\{a_{\beta}: \beta<\Omega\right\}$ of the real line with order type $\Omega$.

For each $n<\omega$, set $a_{n}=\inf E_{n}$. The sequence $\left\langle a_{n}\right\rangle_{n<\omega}$ is then monotone increasing and has infinitely many distinct terms.

For simplicity, assume 0 is a limit ordinal and proceed by transfinite induction on the set of all countable limit ordinals. Let $\beta$ be a countable limit ordinal and suppose for all limit ordinals $\xi<\beta$, a monotone increasing sequence $\left\langle a_{\xi+n}\right\rangle_{n<\omega}$ containing infinitely many distinct terms has been defined with $a_{\xi+n} \in E_{n}$ for all $n$ and $a_{\gamma}<a_{\xi}$ for all $\gamma<\xi$.

There is an increasing sequence $\left\langle\xi_{n}\right\rangle_{n<\omega}$ of ordinal numbers such that $\beta=\sup _{n} \xi_{n},\left\langle a_{\xi_{n}}\right\rangle_{n<\omega}$ is a monotone increasing sequence with infinitely many distinct terms, and $a_{\xi_{n}} \in E_{n}$ for each $n$. For, if there is a largest limit ordinal $\gamma<\beta$, then the desired sequence can be obtained upon setting $\xi_{n}=\gamma+n$. Otherwise, there is an increasing sequence $\left\langle\gamma_{n}\right\rangle_{n<\omega}$ of limit ordinals with $\beta=\sup _{n} \gamma_{n}$ and we set $\xi_{n}=\gamma_{n}+n$.

For each $n$ there is a smallest element $a_{\beta+n} \in E_{n}$ such that $a_{\xi_{m}}<a_{\beta+n}$ for all $m<\omega$. Hence the sequence $\left\langle a_{\beta+n}\right\rangle_{n<\omega}$ is monotone increasing with infinitely many distinct terms, $a_{\beta+n} \in E_{n}$ for all $n<\omega$, and $a_{\gamma}<a_{\beta}$ for all ordinal numbers $\gamma<\beta$.

Thus is defined by transfinite induction a subset $\left\{a_{\beta}: \beta<\Omega\right\}$ of the real line with order type $\Omega$. But the real line has no subset of order type $\Omega$ ! Therefore, the intersection of the sets $E_{n}$ is nonempty.

2. Stationary sequences of sets. In this section we extend a theorem of Zalcwasser [12] on stationary transfinite sequences of ambiguous sets of the first Baire class; i.e., sets which are simultaneously $\mathcal{G}_{\delta}$-sets and $\mathscr{F}_{\sigma}$-sets.

Definition 2. A transfinite sequence $\left\langle E_{\alpha}\right\rangle_{\alpha<\Omega}$ of sets is stationary if there is an ordinal number $\gamma<\Omega$ such that $E_{\alpha}=E_{\gamma}$ for all $\alpha>\gamma$.

THEOREM 2. If $\left\langle E_{\alpha}\right\rangle_{\alpha<\Omega}$ is an ascending or descending transfinite sequence of sets such that for each $\alpha<\Omega$, both $E_{\alpha}$ and its complement are representable as countable unions of ordinally closed sets, then the sequence is stationary.

Proof. We shall apply the reasoning of Zalcwasser, with certain modifications.

Assume $\left\langle E_{\alpha}\right\rangle_{\alpha<\Omega}$ is an ascending transfinite sequence of distinct sets of the stated form. The sets $R_{\alpha}=E_{\alpha+1}-E_{\alpha}$, for $\alpha<\Omega$, are then nonempty disjoint sets and we form a set $T=\left\{t_{\alpha}: \alpha<\Omega\right\}$ by choosing a single point $t_{\alpha}$ from each set $R_{\alpha}$.

The set $P$ of all condensation points in $X$ of the uncountable set $T$ is a perfect set and each point of $P$ is a condensation point of $T \cap P$. Let $D$ be a denumeraije subset of $T \cap P$ which is dense in $T \cap P$, hence also dense in $P$, and which consists of bilateral limit points (i.e., points which are limit points both from the left and from the right) of $T \cap P$; say $D=\left\{t_{\alpha_{n}}: n<\omega\right.$ $\left.\& \alpha_{n}<\Omega\right\}$.

Denoting by $\mu$ the smallest ordinal number $>\alpha_{n}$ for each $n$, we then have 
$D \subset E_{\mu}$. We shall show that both $P \cap E_{\mu}$ and $P-E_{\mu}$ are of the first category in $P$, which yields the contradiction that the perfect set $P=(P \cap$ $\left.E_{\mu}\right) \cup\left(P-E_{\mu}\right)$ is of the first category in itself!

By hypothesis, $E_{\mu}=\cup_{m<\omega} A_{m}$ and $X-E_{\mu}=\cup_{m<\omega} B_{m}$, where the sets $A_{m}$ and $B_{m}$ are ordinally closed for all $m<\omega$. Hence $P \cap E_{\mu}=\cup_{m<\omega}(P \cap$ $\left.A_{m}\right)$ and $P-E_{\mu}=\cup_{m<\omega}\left(P \cap B_{m}\right)$.

We first show each of the sets $P \cap A_{m}$ is nowhere dense in $P$. Suppose $I$ is any open interval with $I \cap P \neq \varnothing$. The set $P-E_{\mu}$ contains all but at most countably many points of the set $T_{\mu}=\left\{t_{\gamma}: \gamma \geqslant \mu\right\}$, and each point of $P$ is a condensation point of $T_{\mu}$; consequently, there is an ordinal number $\alpha \geqslant \mu$ such that $t_{\alpha} \in I \cap\left(P-E_{\mu}\right)$ and $t_{\alpha}$ can also be chosen to be a condensation point of $T_{\mu}$ from both the left and the right. From the inclusion, $P-E_{\mu} \subset P$ - $A_{m}$, we know $t_{\alpha} \notin A_{m}$ and, $A_{m}$ being ordinally closed, there is an open interval $J$, one of whose endpoints is $t_{\alpha}$, which is disjoint from $A_{m}$. Therefore $K=I \cap J$ is an open subinterval of $I, K \cap P \neq \varnothing$, and $K \cap\left(P \cap A_{m}\right)=$ $\varnothing$. Thus $P \cap A_{m}$ is nowhere dense in $P$.

Next we show $P \cap B_{m}$ is nowhere dense in $P$ for each $m<\omega$. Suppose $I$ is any open interval such that $I \cap P \neq \varnothing$. The set $D$ being dense in $P$, there is a point $t_{\alpha_{n}} \in I$. Since $P \cap B_{m} \subset P-E_{\mu}$ and $t_{\alpha_{n}} \notin P-E_{\mu}$, we have $t_{\alpha_{n}} \notin$ $B_{m}$. Hence there is an open subinterval $J$ of $I$, having $t_{\alpha_{n}}$ as an endpoint, such that $J \cap B_{m}=\varnothing$. Since $J \cap P \neq \varnothing$ and $J \cap\left(P \cap B_{m}\right)=\varnothing$, we conclude $P \cap B_{m}$ is nowhere dense in $P$.

By virtue of the contradiction we now obtain, the theorem is true for ascending transfinite sequences.

The case where $\left\langle E_{\alpha}\right\rangle_{\alpha<\Omega}$ is a descending transfinite sequence is obtained from the preceding by complementation.

COROLLARY. Every ascending or descending transfinite sequence of ordinally closed sets is stationary.

Proof. The complement of an ordinally closed set is expressible as a countable union of closed intervals.

As noted by Zalcwasser, his theorem cannot be generalized to arbitrary $\mathcal{G}_{\delta}$-sets (cf. [9], [10]). Theorem 2 does, however, provide a proper extension of his theorem, because there are ordinally closed $\mathcal{G}_{\delta}$-sets which are not $\mathscr{F}_{\sigma}$-sets; the Cantor set minus its left endpoints $\frac{1}{3}, \frac{1}{9}, \frac{7}{9}, \frac{1}{27}, \frac{7}{27}, \frac{19}{27}, \frac{25}{27}, \ldots$ is one such set.

3. Monotone families of sets. A generalization of the Cantor-Baire Intersection Theorem, due to $F$. Riesz [6, p. 21 , footnote], reads: If $\mathscr{N}$ is a nonempty family of compact sets with the property that every finite subfamily of $\mathfrak{N}$ has a nonempty intersection, then $\mathfrak{N}$ has a nonempty intersection.

It is not possible to extend Riesz' theorem to ordinally compact sets, as may be seen upon taking $\mathfrak{K}$ to consist of the sequence of sets $A_{n}=\{x: n /(n$ $+1) \leqslant x<1\} \cup\{n+1\}, n<\omega$. 
In this section we shall, however, extend to ordinally compact sets a corollary of Riesz' theorem which states: If $\mathfrak{N}$ is a monotone family of nonempty compact sets, then there is at least one point in common with all the sets in $\mathfrak{N}$. This corollary, which also provides a generalization of the Cantor-Baire Intersection Theorem, was discovered, independently of Riesz, by W. Sierpiński (see [7]). Riesz' theorem itself was also rediscovered by Sierpiński (see [8]).

DEFINITION 3. A nonempty family $\Re$ of sets is called monotone if for each pair $A, B$ of sets in $\Re$, either $A \subset B$ or $B \subset A$.

THEOREM 3. If $\mathfrak{T}$ is a monotone family of nonempty ordinally compact sets, then the intersection of $\mathfrak{R}$ is nonempty.

Proof. Assume the theorem is false. Then $\mathfrak{N}$, ordered by set-theoretical inclusion, contains no smallest set.

Let $\Theta$ be the smallest ordinal number of power equal to that of $\Re$ and let

$$
A_{0}, A_{1}, \ldots, A_{\alpha}, \ldots, \quad \alpha<\Theta,
$$

be a well-ordering of $\mathfrak{T}$. We shall define by transfinite induction a descending transfinite sequence

$$
E_{0}, E_{1}, \ldots, E_{\alpha}, \ldots, \quad \alpha<\Omega,
$$

of distinct sets in $\Re$.

Set $E_{0}=A_{0}$. Suppose $\alpha<\Omega$ and the sets $E_{\beta}$ have been defined for each ordinal number $\beta<\alpha$.

If $\alpha$ is a successor ordinal, say $\alpha=\gamma+1$, then we define $E_{\alpha}$ to be the first set in (1) which is a proper subset of $E_{\gamma}$.

If $\alpha$ is a limit ordinal, then we first select a denumerable subfamily $\left\{E_{\beta_{n}}: n<\omega\right\}$, with $\beta_{0}<\beta_{1}<\cdots<\beta_{n}<\cdots$, from the sets already defined so that $\alpha=\sup _{n} \beta_{n}$ and, hence, $\bigcap_{n<\omega} E_{\beta_{n}}=\cap_{\beta<\alpha} E_{\beta}$. We know by Theorem 1 that $\cap_{\beta<\alpha} E_{\beta} \neq \varnothing$ and, since $\mathfrak{N}$ is monotone with $\cap \mathfrak{T}=\varnothing$, there must be a set $A \in \mathfrak{N}$ such that $A \subset E_{\beta}$ for all $\beta<\alpha$. We define $E_{\alpha}$ to be the first such set in (1).

Proceeding in this manner, we obtain the transfinite sequence (2), the existence of which contradicts the corollary to Theorem 2 .

\section{REFERENCES}

1. R. Baire, Sur les fonctions de variables réelles, Ann. Mat. Pura Appl. (3) 3 (1899), 1-123.

2. G. Cantor, Contributions to the founding of the theory of transfinite numbers, Dover, New York, 1952.

3. A. Denjoy, L'énumération transfinie, Livre I, Gauthier-Villars, Paris, 1946.

4. I. Grattan-Guinness, The correspondence between Georg Cantor and Philip Jourdain, Jber. Deutsch. Math.-Verein. 73 (1971), 111-130.

5. J. C. Morgan II, Infinite games and singular sets, Colloq. Math. 29 (1974), 7-17.

6. F. Riesz, Stetigkeitsbegriff und abstrakte Mengenlehre, Atti IV Congr. Internaz. Mat., Roma, 1908, Vol. II, pp. 18-24.

7. W. Sierpiński, Uogólnienie pewnego twierdzenia Cantora z teorji mnogości punktowych, Wektor 4 (1915), 49-51. 
8. __ Un théorème sur les ensembles fermés, Bull. Sci. Math. (2) 41 (1917), 290-292 (also appeared in Bull. Intern. Acad. Sci. Cracovie, 1918, 49-51.)

9. __ Sur un ensemble linéaire non dénombrable qui est de première catégorie sur tout ensemble parfait, C. R. Soc. Sci. Varsovie 25 (1932), 102-105.

10. Sur l'existence des suites transfinies décroissantes d'ensembles $F_{\sigma}, \mathbf{C}$. R. Soc. Sci. Varsovie 26 (1933), 85-89.

11. W. H. and G. C. Young, The theory of sets of points, Chelsea, New York, 1972.

12. Z. Zalcwasser, Un théorème sur les ensembles qui sont $\dot{a}$ la fois $F_{0}$ et $G_{\S}$, Fund. Math. 3 (1922), 44-45.

Department of Mathematics, California State Polytechnic University, Pomona, CaliFORNIA 91768 